\title{
Generación de capas de conversión con elementos de tierras raras sobre acero galvanizado
}

\author{
M.A. Arenas* y J.J. de Damborenea*
}

\begin{abstract}
Resumen En el presente trabajo, se desarrollan capas de conversión de cerio sobre un acero galvanizado comercial, proponiéndose un mecanismo de formación de las mismas. Las condiciones óptimas de generación de la capa se consiguieron empleando una disolución de 10.000 ppm de $\mathrm{CeCl}_{3} \cdot 7 \mathrm{H}_{2} \mathrm{O}+0,3 \%$ vol. de $\mathrm{H}_{2} \mathrm{O}_{2}$ ajustando el $\mathrm{pH}$ a 1,3. A su vez, para mejorar la adherencia y reducir el tiempo de tratamiento se realizó, durante los 10 min del tratamiento, una activación potenciostática a $-100 \mathrm{mV}$ respecto al potencial de corrosion, manteniéndose la temperatura a $43^{\circ} \mathrm{C}$. El estudio del corte transversal de las muestras mediante microscopía electrónica de barrido reveló que las capas de cerio poseen un espesor no uniforme de $1-2 \mu \mathrm{m}$. Los resultados electroquímicos indicaron que las capas de cerio generadas ofrecían porcentajes de inhibición del orden del $94 \%$ en cloruro sódico. Un posible mecanismo de formación de la capa de conversión sería que los iones de cerio (III) en solución se oxidan a Ce (IV) por la adición de peróxido de hidrógeno, para precipitar, posteriormente, en forma de óxido de cerio (IV). El potencial catódico aplicado durante la generación de la misma podría originar un proceso de electroreducción de cerio (IV) a cerio (III) en la película de óxido. Por tanto, la capa estaría mayoritariamente compuesta por $\mathrm{Ce}$ (III) encontrándose en menor proporción la presencia de Ce (IV) en la misma.
\end{abstract}

Palabras clave Capas de conversión. Cerio. Inhibidores.

\section{Generation of conversion coatings using rare earths on galvanised steel}

\begin{abstract}
Cerium conversion coatings are formed on a commercial galvanised steel and a generation mechanism is suggested. The best conversion coatings were found using $10,000 \mathrm{ppm}$ $\mathrm{CeCl}_{3} \cdot 7 \mathrm{H}_{2} \mathrm{O}+0,3 \%$ vol. of $\mathrm{H}_{2} \mathrm{O}_{2}$ with a $\mathrm{pH}$ of 1.3 . A cathodic potential, $-100 \mathrm{mV}$ versus corrosion potential, was applied during the treatment at a constant temperature of $43^{\circ} \mathrm{C}$. SEM micrographs have revealed a non uniform coating thickness between $1-2 \mu \mathrm{m}$. Polarization curves in $3.56 \mathrm{wt} . \% \mathrm{NaCl}$ exhibed an inhibition power of about $94 \%$ with respect to the galvanised steel. The proposed mechanism suggests that the Ce (III) ions in the solution were oxidised by $\mathrm{H}_{2} \mathrm{O}_{2}$ to $\mathrm{Ce}$ (IV) which then precipitated as $\mathrm{CeO}_{2}$. The applied voltage could cause a higher presence of $\mathrm{Ce}^{3+}$ in the film maybe due to an electroreduction process of cerium (IV) in the film to cerium (III).
\end{abstract}

Keywords Conversion coatings. Cerium. Inhibitors.

\section{INTRODUCCIÓN}

Una capa de conversión se genera mediante un proceso químico o electroquímico y consiste en la formación de un nuevo óxido con propiedades distintas para sustituir al óxido nativo de la superficie del metal. Se emplea para conferir al material una resistencia extra frente a la corrosión o para mejorar la adherencia de un recubrimiento orgánico con el metal.
Entre los distintos pretratamientos con este objetivo destacan las capas de conversión de cromatos, que se han venido usando de manera general en los pretratamientos anticorrosivos de acabado superficial de los metales utilizados en la industria naval y en la aerospacial.

En el caso del zinc, las primeras capas de conversión de cromatos se empezaron a desarrollar en la década de los años 20. La eficacia en el comportamiento frente a la corrosión de estas capas se

$\left({ }^{*}\right.$ Departamento de Corrosión y Protección. Centro Nacional de Investigaciones Metalúrgicas (CSIC). Avda. Gregorio del Amo 8, 28040 Madrid, 
atribuye a su facultad para actuar como barreras eficaces frente al medio agresivo y a la capacidad de autorreparación de las zonas química o mecánicamente dañadas del recubrimiento por la presencia residual de $\mathrm{Cr}$ (VI) en la película. Este Cr (VI) presente en la capa de conversión lleva a cabo la reparación dinámica de sus defectos y grietas ${ }^{[1}$ y 2$]$. Dicha característica se conoce como protección activa frente a la corrosión y necesita que el agente activo pueda actuar a distancia y que, además, tenga la capacidad de moverse desde la posición "reserva" hasta el sitio dañado.

El principal inconveniente que presentan las capas de conversión de cromatos es la alta toxicidad y la elevada agresividad contra el medioambiente de los compuestos basados en cromo (VI).

Con el objetivo de sustituir las capas generadas con cromatos, en este trabajo, se propone la generación de capas de conversión con sales de cerio en el acero galvanizado, debido a su carácter medioambientalmente limpio y a su posible protección efectiva contra la corrosión. Las capas generadas se estudian desde el punto de vista morfológico y electroquímico, proponiéndose un mecanismo de formación de las mismas.

\section{PARTE EXPERIMENTAL}

Se han generado capas de conversión sobre un acero galvanizado en discontinuo comercial, preparado en un baño de $\mathrm{Zn}$ del $99,99 \%$ de pureza, con $0,01 \% \mathrm{Al}$ y $0,04 \% \mathrm{~Pb}$ a $450{ }^{\circ} \mathrm{C}$ durante $1 \mathrm{~min}$. Las capas de conversión se forman en una disolución de $10.000 \mathrm{ppm} \mathrm{CeCl}_{3} \cdot 7 \mathrm{H}_{2} \mathrm{O}+0,3 \%$ vol. de $\mathrm{H}_{2} \mathrm{O}_{2}$, ajustando el $\mathrm{pH}$ a 1,3. El pH permaneció fijo durante el tratamiento y fue controlado mediante un $\mathrm{pH}$-metro METROHM 691. Durante los 10 min de inmersión en la solución se realiza una activación potenciostática que consiste en la aplicación de un potencial en sentido catódico de -100 $\mathrm{mV}$ respecto al potencial de corrosión. La temperatura durante el tratamiento se mantuvo constante a $43^{\circ} \mathrm{C}$.

El estudio de la morfología de la capa de conversión se llevó a cabo mediante microscopía electrónica de barrido. Para ello se utilizó un equipo JEOL JXA 840 con dos canales de longitud de onda acoplados y un sistema de análisis de dispersión de energía (Energy Dispersive Spectroscopy EDS). En algunas de las muestras se realizó un corte transversal para caracterizar el espesor medio de la capa de conversión mediante un mapping de los distintos elementos presentes en la misma.
Las curvas de polarización se llevaron a cabo usando una celda de tres electrodos, en la que el electrodo de referencia es un electrodo de calomelanos saturado, el contraelectrodo es un hilo de platino y el electrodo de trabajo es el material objeto de estudio. Como medio agresivo se eligió $\mathrm{NaCl} \mathrm{3,56} \mathrm{wt.} \mathrm{\% .} \mathrm{Los} \mathrm{porcentajes} \mathrm{de} \mathrm{inhibición}$ exhibidos por la capa de conversión respecto al galvanizado en este mismo medio se calcularon de acuerdo a la siguiente ecuación:

$$
\operatorname{P.I}(\%)=\frac{\mathrm{i}_{\text {corr }}^{\mathrm{o}}-\mathrm{i}_{\text {corr }}}{\mathrm{i}_{\text {corr }}^{\mathrm{o}}} \times 100
$$

donde, $\mathrm{i}_{\text {corr }}^{\mathrm{o}}$ e $\mathrm{i}_{\text {corr }}$ son las densidades de corriente de corrosión sin y con inhibidor, respectivamente.

\section{RESULTADOS Y DISCUSIÓN}

\subsection{Condiciones de generación de la capa}

En este apartado se explica detalladamente la elección de cada uno de los parámetros de generación de la capa de conversión de cerio.

La concentración de cerio se fijó en 10.000 ppm ya que, a concentraciones más pequeñas de $\mathrm{CeCl}_{3} \cdot 7 \mathrm{H}_{2} \mathrm{O}$ en la solución aparecían zonas desnudas sobre el sustrato, creándose en algunas ocasiones un polvillo amarillento poco adherente sobre la superficie.

La aplicación del tratamiento potenciostático mejoró la adherencia de la capa respecto a aquellas generadas por simple inmersión en el medio. A su vez, permitió disminuir el tiempo tratamiento. Este hecho se debe a que el cerio ve favorecida su cinética de precipitación en forma de óxido/hidróxido al obligar al material a que actúe de cátodo durante el tratamiento. Sobre la superficie se generan una gran cantidad de grupos $\mathrm{OH}^{-}$acelerándose, por tanto, la generación de la capa. De todos los potenciales estudiados, los mejores resultados se encontraron aplicando un potencial de $-100 \mathrm{mV}$ frente al potencial de circuito abierto.

En cuanto a la elección del $\mathrm{pH}$, la solución con 10.000 ppm de $\mathrm{CeCl}_{3}$ presenta un $\mathrm{pH}$ de $4,6 \mathrm{y}$, al añadir el $\mathrm{H}_{2} \mathrm{O}_{2}$, éste vira hasta un valor de 3,2 , aproximadamente. Para que se lleve a cabo la deposición de una capa de conversión, el $\mathrm{pH}$ de la solución debe originar la disolución del sustrato metálico a la vez de permitir la formación de la película. En el caso del zinc, a pH menores de 4, su velocidad de disolución alcanza un valor apreciable según la reacción: 


$$
\mathrm{Zn}+2 \mathrm{H}^{+} \rightarrow \mathrm{Zn}^{2+}+\mathrm{H}_{2}^{\uparrow}
$$

Por tanto, como al añadir el peróxido de hidrógeno, el $\mathrm{pH}$ de la solución se sitúa en un valor de 3,2 , se garantizaría la primera de las premisas para que se lleve a cabo la deposición de la capa de conversión. Al estudiar las propiedades de la capa generada a este $\mathrm{pH}$ se obtuvo que dichas capas no presentan una buena adherencia al sustrato, desprendiéndose en algunas zonas. Con el objetivo de mejorar la adherencia de la capa se ajustó el pH a valores más pequeños, llegándose a encontrar un $\mathrm{pH}$ óptimo de generación de la capa en un valor de 1,3. Este comportamiento es análogo al observado para la generación de capas de cromatos sobre zinc, aluminio y cadmio, donde el $\mathrm{pH}$ debe estar comprendido entre 1 y 2 para que la capa exhiba una buena calidad ${ }^{[3]}$.

La adición del $\mathrm{H}_{2} \mathrm{O}_{2}$ a la solución, además de disminuir el $\mathrm{pH}$ de la misma, provoca que el cerio presente en la solución acuosa en forma de iones hidratados de $\mathrm{Ce}^{3+}$ se oxide a $\mathrm{Ce}^{4+}$, favoreciendo de esta forma la incorporación de cerio en estado (IV) en la capa de conversión.

Por último, el aumento de temperatura a $43^{\circ} \mathrm{C}$ respecto a la temperatura ambiente, acelera la reacción de formación de la capa de conversión al favorecer la cinética de deposición del cerio. Para temperaturas superiores a este rango se comprobó que no se generaban capas continuas y uniformes sobre la superficie.

\subsection{Caracterización de la capa de conversión}

En la figura 1 se muestra una micrografia SEM de la capa de conversión de sales de cerio generada sobre el acero galvanizado. La espectroscopía por dispersión de energías de la superficie reveló la presencia de cerio, oxígeno, cloro y zinc como principales componentes de la misma (Fig. 2).

Esta composición fue corroborada por los análisis de espectroscopia fotoelectrónica de rayos X, XPS ${ }^{[4]}$. Dichos análisis indicaron que la capa de conversión estaba compuesta principalmente en su zona más externa por una mezcla de un óxido hidratado/hidróxido de $\mathrm{Ce}^{3+}$ y $\mathrm{Ce}^{4+}$ junto con un óxido de zinc, $\mathrm{ZnO}$, y en su parte más interna, por un óxido de cerio, mayoritariamente $\mathrm{Ce}_{2} \mathrm{O}_{3}$, y $\mathrm{ZnO}$.

La figura 3 permite concluir que el espesor de la capa de conversión es del orden de $1-2 \mu \mathrm{m}$ y no es uniforme a lo largo de toda la superficie.

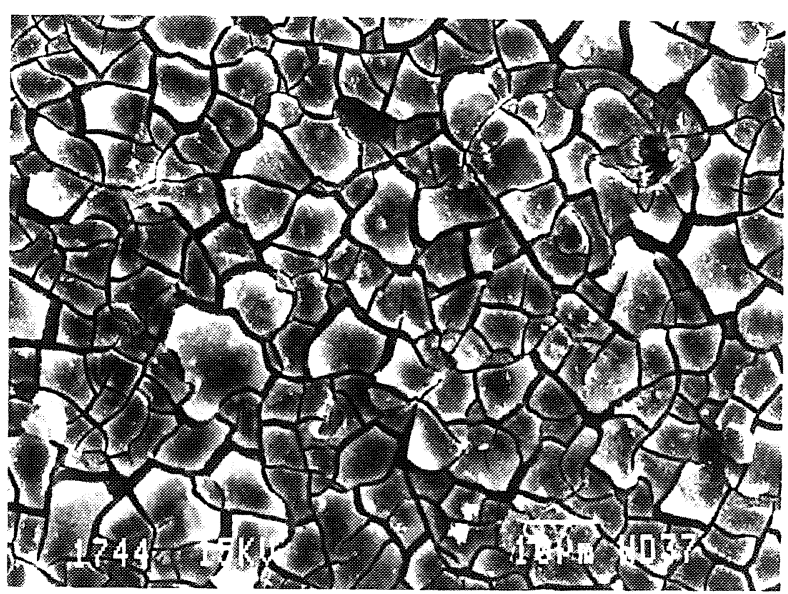

Figura 1. Micrografia SEM de la capa de conversión.

Figure 1. SEM image of the conversion coating.

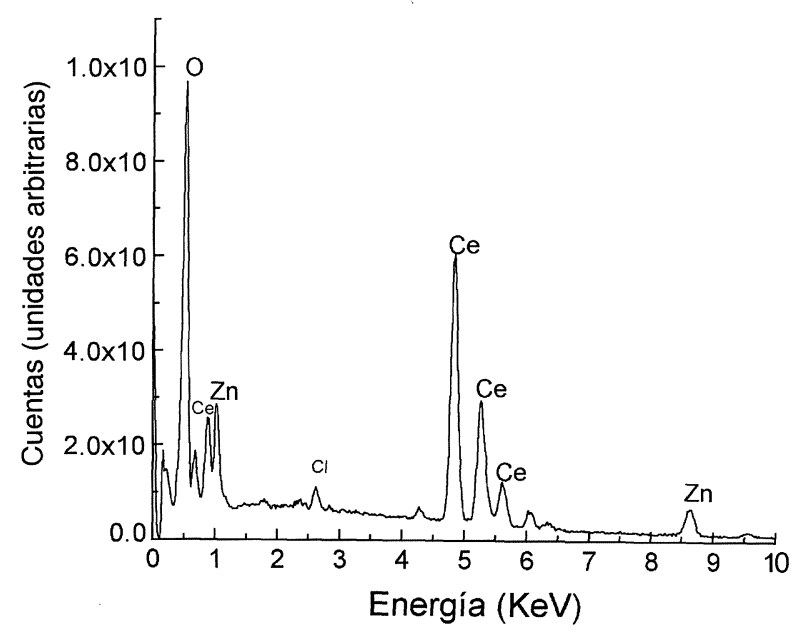

Figura 2. EDS de la capa de conversión.

Figure 2. EDS analysis of the cerium conversion coating.

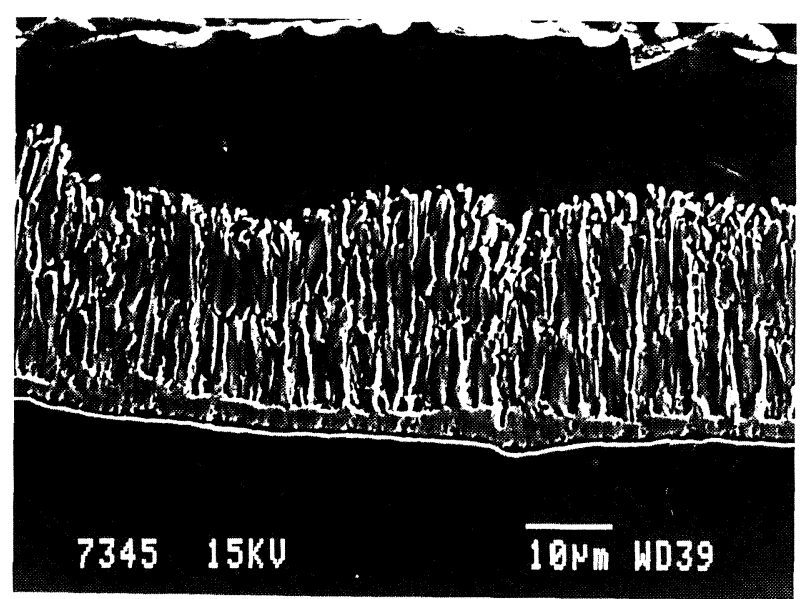

Figura 3. Corte transversal del acero galvanizado tras la generación de la capa de conversión. El espesor de la misma es de $1-2 \mu \mathrm{m}$.

Figure 3. Transversal section of the galvanised steel with the cerium conversion coating on the top. The thickness of the coating is about $7-2 \mu \mathrm{m}$. 


\subsection{Caracterización electroquímica}

En la figura 4 se muestra la curva de polarización lineal de la capa de conversión en $\mathrm{NaCl}$ 3,56 wt. \% después de 15 min de inmersión. A modo de comparación, se incluyen, en esta misma figura, las curvas correspondientes al acero galvanizado en $\mathrm{NaCl}$ 3,56 wt. \%, en una solución de cloruro sódico con $10.000 \mathrm{ppm} \mathrm{CeCl}_{3} \cdot 7 \mathrm{H}_{2} \mathrm{O}$ y la de una capa de conversión generada en una solución de 10.000 ppm de $\mathrm{CeCl}_{3} \cdot 7 \mathrm{H}_{2} \mathrm{O}+0,3 \%$ vol. $\mathrm{H}_{2} \mathrm{O}_{2}$, sin ajustar los otros parámetros de generación. Como se observa en la gráfica, las muestras con la capa de conversión presentan los valores de densidad de corriente de corrosión más pequeños. Además, la forma de las curvas revela un cambio en el mecanismo que controla el proceso de corrosión: se pasa de un control catódico a un control por activación.

Los valores en porcentajes de inhibición exhibidos por las capas de conversión son de un $94 \%$ de protección respecto al galvanizado en cloruro sódico.

\subsection{Mecanismo de formación de la capa de conversión}

Para proponer un posible mecanismo de formación de la capa es necesario estudiar bajo las condiciones experimentales usadas, cuáles son los procesos de precipitación de las especies de cerio en solución implicados.

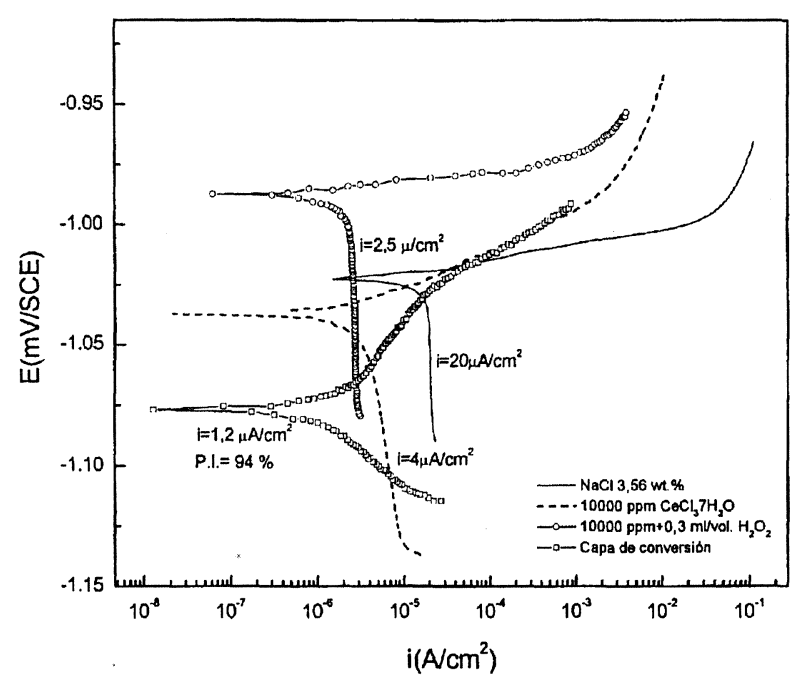

Figura 4. Curvas de polarización tras 15 min de inmersión en $\mathrm{NaCl} 3,56$ wt. \%.

Figure 4. Polarization curves after $15 \mathrm{~min}$ in $\mathrm{NaCl} 3.56$ wt. \%.
La adición de peróxido de hidrógeno en el medio provoca un viraje de color en el mismo, de transparente a amarillo, y una disminución del $\mathrm{pH}$ de 4,6 a 3,2. El paso de los iones $\mathrm{Ce}^{3+}$ a $\mathrm{Ce}^{4+}$ en solución es el responsable de este viraje de color observado ya que el $\mathrm{Ce}^{4+}$ es de tonalidad amarillaanaranjada y el $\mathrm{Ce}^{3+}$ es transparente y la acidificación del medio se debe al proceso redox implicado:

$$
\begin{gathered}
\mathrm{H}_{2} \mathrm{O}_{2}+2 \mathrm{H}^{+}+2 \mathrm{e}^{-} \rightarrow 2 \mathrm{H}_{2} \mathrm{O} \\
\mathrm{Ce}^{3+}+2 \mathrm{H}_{2} \mathrm{O} \rightarrow \mathrm{Ce}(\mathrm{OH})_{2}{ }^{2+}+2 \mathrm{H}^{+}+\mathrm{e}^{-}
\end{gathered}
$$

siendo la reacción redox global:

$2 \mathrm{Ce}^{3+}+2 \mathrm{H}_{2} \mathrm{O}+\mathrm{H}_{2} \mathrm{O}_{2} \rightarrow 2 \mathrm{Ce}(\mathrm{OH})_{2}{ }^{2+}+2 \mathrm{H}^{+}$

A continuación, se estima si el peróxido añadido, 0,3 \% en volumen, es suficiente para oxidar todo el cerio (III) en solución a cerio (IV). Como la solución es de $10.000 \mathrm{ppm} \mathrm{CeCl}_{3}$ y, si por ejemplo, suponemos un volumen total de $50 \mathrm{ml}$, tenemos que la cantidad de cerio a añadir es de 0,5 gramos ó $1,3419 \cdot 10^{-3}$ moles de $\mathrm{CeCl}_{3} \cdot 7 \mathrm{H}_{2} \mathrm{O}$, que a su vez serían los moles de cerio. Como por la estequiometría de la reacción, un mol de cerio reacciona con $1 / 2$ de peróxido, entonces para oxidar los moles de cerio contenidos en los 10.000 ppm necesitaríamos $6,7095 \cdot 10^{-4}$ moles de $\mathrm{H}_{2} \mathrm{O}_{2}$. Teniendo en cuenta que la densidad del peróxido de hidrógeno es de $1,1 \mathrm{~g} / \mathrm{cm}^{3}$, el volumen de $\mathrm{H}_{2} \mathrm{O}_{2}$ necesario sería:

$$
V=m \cdot d=0,0250 m l
$$

si el $\mathrm{H}_{2} \mathrm{O}_{2}$ fuera del $100 \%$ de pureza. Como para generar la capa de conversión añadimos el 0,3\% vol. de $\mathrm{H}_{2} \mathrm{O}_{2}$, es decir, $0,15 \mathrm{ml} \mathrm{de} \mathrm{H}_{2} \mathrm{O}_{2}$ al $100 \%$ de pureza, estamos, por tanto, añadiendo más cantidad de peróxido a la solución, lo que garantiza que todo el cerio (III) en el medio está en forma de $\mathrm{Ce}(\mathrm{OH})_{2}{ }^{2+}$.

Este hecho nos permite estimar el $\mathrm{pH}$ necesario para la precipitación del $\mathrm{CeO}_{2}$ a partir de los iones $\mathrm{Ce}(\mathrm{OH})_{2}{ }^{2+}$ mediante la siguiente ecuación:

$$
\log \left[\mathrm{Ce}(\mathrm{OH})_{2}{ }^{2+}\right]=19,22-2 \mathrm{pH}
$$

ya que se puede asumir que todos los iones Ce (III) en solución (10.000 ppm) se han oxidado por la adicción del $\mathrm{H}_{2} \mathrm{O}_{2}$ y están en forma $\mathrm{Ce}(\mathrm{OH})_{2}{ }^{2+}$. Al sustituir dicha concentración en (6) se obtiene que el valor del $\mathrm{pH}$ de precipitación es de 10,39. Por tanto, únicamente cuando la reacción de 
reducción del $\mathrm{O}_{2}$ sea vía un proceso de 2 electrones en las zonas catódicas se alcanzaría un $\mathrm{pH}$ que permite la precipitación del oxido de cerio (IV) ${ }^{[5]}$. Este proceso, termodinámicamente posible, fue favorecido cinéticamente al añadir peróxido de hidrógeno en el medio para que actuase como agente acelerador en la formación de las capas de conversión con sales de cerio. El $\mathrm{H}_{2} \mathrm{O}_{2}$ es un oxidante más eficaz en el proceso de precipitación del óxido de cerio que el $\mathrm{O}_{2}$, porque tanto la concentración de $\mathrm{OH}^{-}$como la de $\mathrm{HO}_{2}^{-}$exhiben un gradiente de concentración en la interfase metal/electrolito semejante, a diferencia de lo que sucede entre el $\mathrm{O}_{2}$ y los $\mathrm{OH}^{-}$que lo presentan opuesto.

Bajo estas condiciones experimentales, el mecanismo propuesto por Aldykewicz ${ }^{[6]}$ para la formación de un óxido de cerio (IV) a partir de la precipitación de los iones acuosos $\mathrm{Ce}(\mathrm{OH})_{2}{ }^{2+}$ puede explicar la presencia de cerio (IV) en las capas, de acuerdo con la composición revelada en los espectros de XPS ${ }^{[5]}$.

El porcentaje de cerio (IV) esperado en la capa podría modificarse por la aplicación del potencial catódico para mejorar la adherencia de la misma al sustrato. La polarización catódica originaría un proceso de electroreducción del cerio (IV) a cerio (III) en la película de óxido,

$$
\mathrm{Ce}^{4+}+\mathrm{e}^{-} \rightarrow \mathrm{Ce}^{3+}
$$

lo que sugeriría una presencia mayoritaria de cerio (III) en la capa, permitiendo explicar los resultados de los análisis de XPS, según los cuales, la capa de conversión estaría compuesta mayoritariamente en su zona interna por un óxido de cerio (III). Además, la presencia de cerio (III) de forma predominante en la película al aumentar el tiempo de sputtering, podría deberse a la reducción del cerio (IV) a cerio (III) por el efecto del bombardeo iónico con argón o por el daño sufrido por la acción de los rayos $\mathrm{X}^{[7-9]}$.

\section{CONCLUSIONES}

Las capas de conversión con sales de cerio generadas en el acero galvanizado comercial presentan un espesor no uniforme de $1-2 \mu \mathrm{m}$ y están compuestas por una mezcla de óxido de Ce (III) y Ce (IV).

La forma de la curva de polarización en cloruro sódico revela un cambio en el mecanismo de corrosión del sistema: se pasa de un control por difusión a un control por activación, proporcionándose un porcentaje de inhibición del $94 \%$.

El mecanismo de formación de la capa de conversión propuesto en el presente trabajo considera que todos los iones de cerio (III) en solución se oxidan a Ce (IV) por la adición de peróxido de hidrógeno. Posteriormente, los iones de $\mathrm{Ce}^{4+}$ en solución precipitan en forma de óxido de cerio (IV) explicando la presencia de cerio (IV) en la capa. Por otro lado, el potencial catódico aplicado durante la generación de la misma podría originar un posible proceso de electroreducción del cerio (IV) a cerio (III) en la capa de óxido, explicando la presencia mayoritaria de cerio (III) en la misma.

\section{Agradecimientos}

Este proyecto se ha financiado gracias al proyecto del Plan Nacional de I+D MAT99-0625-C02-02. "Alternativas al cromo para el acabado superficial de aleaciones de aluminio".

\section{REFERENCIAS}

[1] M.W. Kendig, A.J. Davenport y H.S. IsaAcs, Corr. Sci. 34 (1993) 41-49.

[2] F.W. Lytle, R.B. Greegor, G.L. Bibbins, K.Y. Blohowiak, R.E. Smith y G.D. Tuss, Corr. Sci. 37 (1995) 349-369.

[3] K.A. KorineK, Corrosion Protection Methods, pp. 389-395.

[4] M.A. Arenas y J. De Damborenea, $15^{\text {th }}$ Int. Corros. Congr., ICC, Granada, Spain, 2002.

[5] M.A. Arenas y J. De Damborenea, Electrochim. Acta 48 (2003) 3.693-3.698.

[6] A.J. Aldykiewiczs JR., A.J. Davenport y H.S. IsaAcs, J. Electroch. Soc. 143 (1996) 147-153.

[7] A.E. Hughes, J.D. Gorman, P.J.K. Patterson y R. CARTER, Surf. Interface Anal. 24 (1996) 634-640.

[8] E. Paparazzo, Surf. Sci. Lett. 234, L.253 (1990).

[9] J. El Fallah, L. Hilaire, M. Roméo y F. Le Normand, J. Electron Spectrosc. Relat. Phenom. 73 (1995) 89-103. 